\title{
Detection of the Change in Mitochondrial Membrane Potential at the Cone-shaped Projection in Nephron Morphogenesis.
}

\author{
M. Nishikawa, S. Morikawa, K. Shimizu, T. Ezaki
}

Tokyo Women's Medical University, Tokyo, 162-8666, Japan

It is known that mesenchymal cells of the metanephric mesoderm interact with the ureteric duct which is important in the morphogenesis of the nephron. Although it is recognized that the mesenchymal cells form a cone-shaped projection[1], the mechanism by which this connects with the ureteric duct is not fully understood. In this study, we focused the change in mitochondrial membrane potential at the cone-shaped projection related to proximal tubular morphogenesis in rat fetal kidney and in cultured LLC-RK1(rat proximal cell line).

Electron microscopy; Tissue specimens of the embryonic kidneys were taken from 18 dpc Wistar rat embryo. In this age, the cortex of the kidney contains immature nephrons. The specimens were fixed in $2 \%$ glutaraldehyde, post fixed with $1 \%$ OsO4, embedded in epoxy resin, and examined by electron microscopy.

Immunohistochemistry; Tissue specimens were divided into $40 \mu \mathrm{m}$ sections, fixed with $4 \%$ paraformaldehyde in $0.1 \mathrm{M}$ phosphate-buffered saline (PBS) and $30 \%$ sucrose.

Immunohistochemical staining was performed using rhodamin-conjugated anti-cytochrome $\mathrm{C}$ antibody and examined by a confocal laser scanning microscopy (Leica).

Detection of the change in mitochondrial membrane potential; ApoAlelert ${ }^{\mathrm{TM}}$ Mitochondria Membrane Sensor Kit was employed for the detection of mitochondrial membrane potential in LLC-RK1 cells (rat proximal cell line). In the intact state, a cationic lipophilic dye (rhodamine 123) partitions across the inner membrane based on charge showing red [2]. In the apoptotic cells, the rhodamine 123 indicates hyperpolarization of the inner membrane, leading release of cytochrome $\mathrm{C}$ and non-aggregated dye shows green.

Apoptotic bodies appeared at the cone-shaped junction near the ureteric duct (Fig. 1A). These cells showed normal patterns of chromatin distribution but contained 2 or 3 nucleoli and exhibited an elevated euchromatin:heterochromatin ratio. The cytoplasm contained rough endoplasmic reticulum. The view of this area showed several cells that were either apoptotic or contained apoptotic bodies. Nuclear fragmentation was evident, and within the S-shaped renal vesicles that were continuous with the ureteric duct, cells demonstrated condensed chromatin. An arrow indicates the transitional area between renal vesicle and ureteric duct (Fig. 1A). Collecting duct clear cells were apparent among the dark intercalated cells. The left -center of Fig.1A shows a cell, containing a crescent-like arrangement of chromatin. Apoptotic bodies were seen at the side of the ureteric duct and macrophage phagocytosis was also evident. The hypermagnification in apoptotic area disclosed that some mitochondria showed irregular shaped membrane (Fig. 1B). Some of the sheeting LLC-RK1 cells formed dome like structure resembling ureteric duct. Cells with intact mitochondria disclosed dye aggregation stained red as keeping mitochondrial membrane potential. However cells in which membrane potential was broken were stained green (Fig. 2). Cytochrome $\mathrm{C}$ was not stained in an apoptotic cell (Fig.3A,B).

These findings suggest that mitochondrial membrane potential may be important and involved in apoptosis during nephron morphogenesis, especially at the cone-shaped projection of the developing kidney .

Reference

[1] N. Iino et al., Arch.Histol 64(2001) 179.

[2] D.R. Green et al., Science 281 (1998) 1309. 


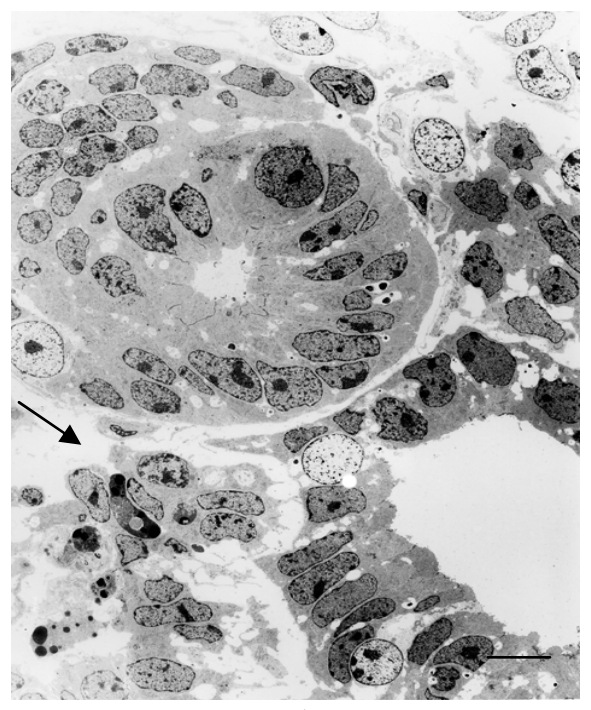

A

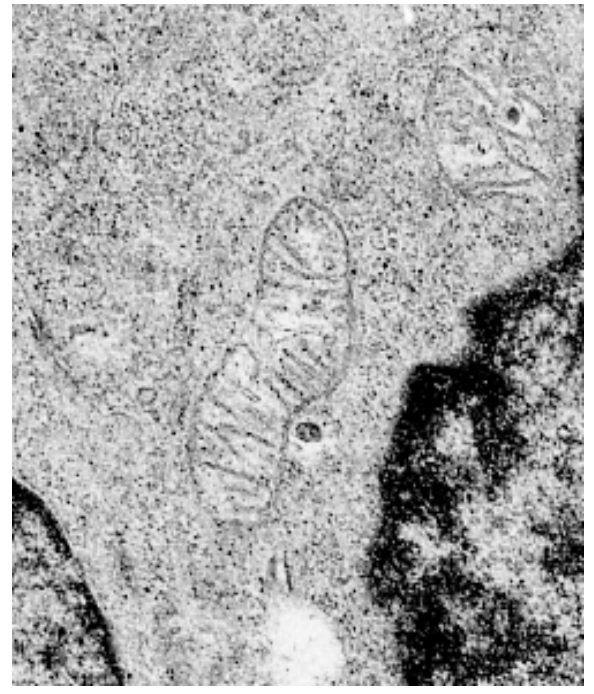

B

FIG. 1.A )Electron microscopic findings of metanephros of $18 \mathrm{dpc}$ fetal rat. Apoptotic cell death is disclosed at the connecting area between renal vesicle and ureteric duct (arrow). Bar $=1 \mu \mathrm{M}$. B) The hypermagnification of mitochondria in apoptotic area. Some mitochondria show irregular shaped membrane. Bar $=5 \mu \mathrm{M}$.

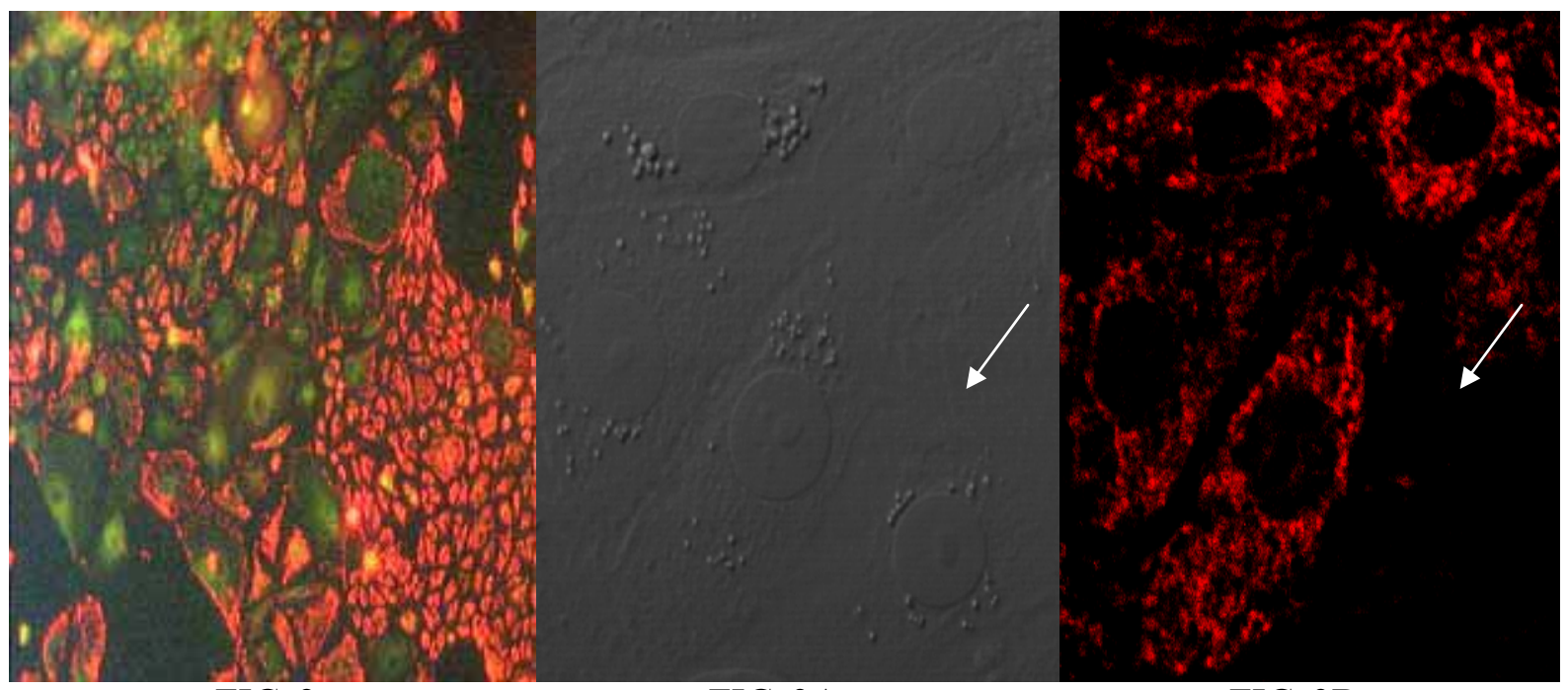

FIG. 2

FIG. 3A

FIG. 3B

FIG. 2. Some of the sheeting LLC-RK1 cells are forming dome like structure resembling ureteric duct. Cells with intact mitochondria show dye aggregation stained red as keeping mitochondrial membrane potential. However cells in which membrane potential is broken are stained green (X 100).

FIG. 3. A) The sheeting LLC-RK1 cells (phase appearance). B) Cytochrome $\mathrm{C}$ staining of LLC-RK1 cells. A cell (lower right) shows loss of cytochrome $\mathrm{C}$ staining (X 400). 